\title{
Study of Forced Convection in a Pipe Partially Filled With a Porous Medium
}

\author{
HABIBOLLAH SAYEHVAND and HOSSEIN SHOKOUHMAND \\ Department of Mechanical Engineering, Bu-Ali Sina University, Hamadan, and Department of Mechanical Engineering, \\ University of Tehran, Tehran \\ P.O.Box: 65175-4161, Hamadan, \\ IRAN
}

\begin{abstract}
The objective of the present work was to perform a detailed numerical study of laminar fully developed forced convection in a pipe partially filled with a porous medium. The Brinkman-Forchheimerextended Darcy model is adopted for the porous matrix region, which is attached at the pipe wall and extends inward, toward the centerline. The effects of the Darcy number $D a$, the thickness of porous layer $S$ and the thermal conductivity ratio, $R_{k}$, are investigated. The results showed that as thickness of the porous layer increases or the Darcy number decreases, the pressure drop grows. It was found that, the dependence of the Nusselt number $N u$ on $S$ is not straightforward. For a higher thermal conductivity porous material, the Nusselt number increases monotonically with increasing $S$ while for a lower value of $R_{k}$ a minimum exists in the $N u$ distributions. The obtained results show that increasing either the Darcy number or the thermal conductivity of porous material improves the heat transfer. Further, for highly permeable and conducting porous media, it may not be necessary to fill the pipe cross section completely to attain the maximum heat transfer.
\end{abstract}

Key-Words: - Forced convection, Nusselt, Porous medium, Pressure Drop, SIMPLER, Thickness,

\section{Introduction}

Fundamental studies of thermal convection in porous media have increased significantly during recent years. Such a strong interest stems from the various practical applications involving porous media such as compact heat exchangers, geothermal systems, thermal insulation, energy storage units, ceramic processing, packed bed chemical reactors, and heat transfer enhancement which has received significant recent interest especially in high heat flux environments. Extensive reviews of flow and heat transfer in porous media were provided by Nield and Bejan [1] and Kaviany[2].

An important class of problems directly related to porous matrix convection is heat and fluid flow in composite systems, that is, systems consisting partly of a fluid-saturated porous material and partly of a fluid. The convection phenomenon in these systems is usually affected by the interaction of the temperature and flow fields in the porous spaces and the open spaces. The importance of this class of problem is justified both in a fundamental and in a practical sense. With reference to practical thermal Engineering applications which stand to benefit if a better understanding of heat and fluid flow processes in composite systems is acquired, the following examples are cited: fibrous and granular insulationwhich occupies only part of the space between a hot and a cold boundary, fault zones in geothermal systems, the cooling of stored grain, and heat removal from nuclear debris beds in nuclear reactor safety.

Most of the existing studies on convection in composite layers focus on the problem of natural convection in an enclosure [3] or an infinite horizontal layer partially filled with a porous material (see for example, [4] and [5]). Forced convection in a composite system, in which a fluid-saturated porous material occupies only a part of a confined passageway, has been the topic of several investigations published in the literature since the work of Poulikakos and Kazmierczak [6]. An important finding was made by Tong and Sharachandra [7] that the use of only a slice of porous material inserted in a channel could give rise to considerably augmentation of convective heat transfer. An enhanced heat transfer in an annular duct partially filled with a porous medium with high permeability and conductivity was observed in a recent analytical solution of Chikh et al. [8] with the Brinkman model. Sung et al. 
[9] simulated numerically the forced convection from an isolated heat source in a channel, in which the loss of pressure drop was reduced by employing a partiallyporous channel. Recently, Guo et al. [10] simulated the pulsating flow and heat transfer in a pipe partially filled with a porous medium.

In the present treatise, the Brinkman-Forchheimerextended Darcy model is employed for the region of porous medium. Efforts are focused on identifying the influence of non-dimensional parameters on flow and heat transfer properties, such as the Darcy number, the thickness of porous layer and the thermal conductivity ratio. By numerically solving the governing equations, the details of both flow and thermal fields are acquired. The numerical results are expected to provide an improved understanding of the underling physical phenomena. This study also aims to construct a baseline framework for predicting heat transfer and flow features of more complex configurations. This aspect is significant in view of the lack of documented experimental data on heat transport in a pipe partially filled with a porous medium.

\section{The Model}

Consider a pipe of radius $a$ which partially filled with a fluid-saturated porous medium, as sketched in Fig.1. An entrance region of length $L_{1}$ exists at the inlet, this is followed by the region of length $L_{2}$ in which both the temperature and the flow fields are assumed to be fully developed. The porous matrix of thickness $s$ is attached at the pipe wall and extends inward, toward the centerline. Due to the symmetry with respect to the centerline of the pipe, the calculation domain was limited to a half-width of flow pipe in this study. In conformity with the majority of preceding studies, the porous medium is taken to be homogeneous, isotropic and in local equilibrium with the fluid. The thermophysical properties of the fluid and the effective properties of the porous medium are assumed to be constant $[11,12]$.

With the above assumptions, the time-dependent governing equations, in properly non-dimensionalized form, can be written as, using standard notation $[11,12$, 13]:

$\frac{\partial U}{\partial X}+\frac{1}{R} \frac{\partial}{\partial R}(R V)=0$

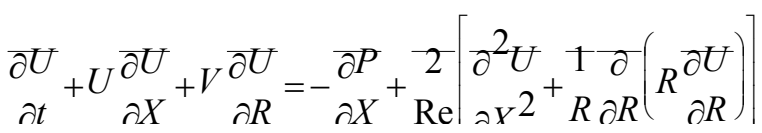

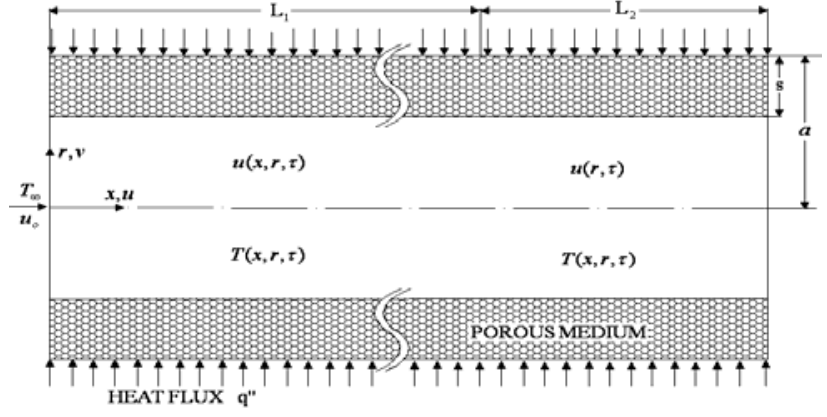

Fig.1. Schematic diagram of heated circular pipe partially filled with a porous medium.

$-\varsigma\left[\frac{2 \varepsilon}{D a \operatorname{Re}}+\frac{\varepsilon^{2} C_{F}}{\sqrt{D a}}\left|\overrightarrow{V_{D}}\right|\right] U$

$\frac{\partial V}{\partial t}+U \frac{\partial V}{\partial X}+V \frac{\partial V}{\partial R}=-\frac{\partial P}{\partial R}+\frac{2}{\operatorname{Re}}\left[\frac{\partial^{2} V}{\partial X^{2}}+\frac{1}{R} \frac{\partial}{\partial R}\left(R \frac{\partial V}{\partial R}\right)-\frac{V}{R^{2}}\right]$

$-\varsigma\left[\frac{2 \varepsilon}{D a \operatorname{Re}}+\frac{\varepsilon^{2} C_{F}}{\sqrt{D a}}\left|\overrightarrow{V_{D}}\right|\right] V$

$R_{c} \frac{\partial \Theta}{\partial t}+U \frac{\partial \Theta}{\partial X}+V \frac{\partial \Theta}{\partial R}=\frac{2\left\lfloor 1+\left(R_{k}-1\right) \zeta\right]}{\varepsilon \operatorname{Re} \operatorname{Pr}}$

$\left[\frac{\partial^{2} \Theta}{\partial X^{2}}+\frac{1}{R} \frac{\partial}{\partial R}\left(R \frac{\partial \Theta}{\partial R}\right)\right]$

in which $\left|\vec{V}_{D}\right|=\sqrt{U^{2}+V^{2}}, U$ and $V$ represent the fluid

velocity components in the fluid region as well as the Darcian velocity components in the porous medium region, $\varepsilon$ denotes the porosity, $C_{F}$ the inertia coefficient of the porous medium and $\operatorname{Pr}$ the Prandtl number. The above formulations are applicable to both fluid and porous regions; the fluid occupied region is identified by setting the index $\varsigma=0$ and the porous region tagged by $\varsigma=1$. The momentum equations represent the Brinkman-Forchheimer-extended Darcy model [11], which includes both viscous and inertia effects. It is noted that the last terms in equations (2) and (3) indicate an improved model to account for the inertia effect in the momentum equations. Equation (4) is the energy equation, which is widely utilized to tackle timedependent convective processes in porous media.

In the above, the non-dimensional quantities are defined as:

$$
X=\frac{x}{a}, \quad R=\frac{r}{a}, \quad U=\frac{u}{\varepsilon u_{0}}, \quad V=\frac{v}{\varepsilon u_{0}},
$$


$t=\frac{\tau}{a / u_{0}}, \quad P=\frac{p}{\rho u_{0}^{2}}, \quad \Theta=\frac{T}{q^{\prime \prime} a / k}$,

where $u_{0}$ denotes the unidirectional velocity of constant temperature $T_{\infty}$ at the inlet. Further, the non-dimensional thickness of the porous medium is $S=s / a$. The dimensionless parameters in equations (1)-(4) are defined as:

Reynolds number: $\operatorname{Re}=\frac{2 a u_{0}}{v}$

Darcy number: $D a=\frac{K}{a^{2}}$

Prandtl number:

$$
\operatorname{Pr}=\frac{v}{\alpha}
$$

Thermal conductivity ratio: $R_{K}=\frac{k_{e f f}}{k}$

Heat capacity ratio: $\quad R_{c}=\frac{\left(\rho C_{p}\right)_{e f f}}{\varepsilon\left(\rho C_{p}\right)}$

Where $v$ is the kinematic viscosity, $\alpha$ the thermal diffusivity, and $k$ the thermal conductivity of he fluid, while $k_{\text {eff }}$ is the effective thermal conductivity and $K$ the permeability of the porous medium.

In the present paper, the porosity $\varepsilon$ of the porous medium is set to be 0.6 and the inertia coefficient $C_{F}$ in Forchheimer's extension is taken to be equal to 0.057 in accordance with the previous works $[14,15]$. This value is admittedly too low for conventional sphere-shaped porous material for which the Ergun relation [2] is applicable. However, for Foametal, which is gaining popularity in advanced heat-exchanger application, $C_{F}$ takes a much smaller value. The present account is addressed to these applications. While the precise value of $v_{\text {eff }}$ in Brinkman's extension remains somewhat unclear, $v_{e f f}$ is taken to be the same as $v$, as a first approximation as ascertained by Lundgren [16].

To solve the fully developed forced convection, the flow field in the entrance region must be obtained beforehand. Accordingly, the associated boundary conditions are formulated as follows. At the pipe inlet (at $X=0$, for $0<R<1$ ):

$U(0, R, t)=1$,

$V(0, R, t)=0$.

At the pipe wall $\left(R=1\right.$, for $\left.0<X<L_{1}\right)$ :

$U(X, 1, t)=0$,

$V(X, 1, t)=0$.

Owing to the symmetry requirement at the pipe centerline (at $R=0$, for $0<X<L_{1}$ ), it follows that:

$\frac{\partial U}{\partial R}(X, 0, t)=0$,

$V(X, 0, t)=0$,

At the end of region of length $L_{1}$, the flow is assumed to have attained a fully developed state. This is a customary procedure invoked under the assumption that the pipe is sufficiently long, i.e. $L_{1}>>1$. Accordingly, at $X=L_{1}$, for $0<R<1$ :

$\frac{\partial U}{\partial X}\left(L_{1}, R, t\right)=0$,

$\frac{\partial V}{\partial X}\left(L_{1}, R, t\right)=V\left(L_{1}, R, t\right)=0$,

For the fully developed region, the momentum and energy equations are written as:

$$
\begin{aligned}
& \frac{\partial U}{\partial t}=-\frac{\partial P}{\partial X}+\frac{2}{\operatorname{Re}} \frac{1}{R} \frac{\partial}{\partial R}\left(R \frac{\partial U}{\partial R}\right) \\
& -\varsigma\left[\frac{2 \varepsilon}{D a \operatorname{Re}}+\frac{\varepsilon^{2} C_{F}}{\sqrt{D a}}|U|\right] \\
& R_{c} \frac{\partial \Theta}{\partial t}+U \frac{\partial \Theta}{\partial X}=\frac{2\left[1+\left(R_{k}-1\right) \varsigma\right]}{\varepsilon \operatorname{Re} \operatorname{Pr} R} \frac{\partial}{\partial R}\left(R \frac{\partial \Theta}{\partial R}\right)
\end{aligned}
$$

The boundary conditions are as follows:

$U(X, 1, t)=0$,

$\frac{\partial \Theta}{\partial R}(X, 1, t)=\frac{1}{R_{k}}$,

$\frac{d U}{d R}(X, 0, t)=0$,

$\frac{\partial \Theta}{\partial R}(X, 0, t)=0$.

To solve the above system of equations, Chatwin's approximation is employed [17], i.e.

$\frac{\partial \Theta}{\partial X}=\frac{4}{\operatorname{RePr}}=$ const.

\section{Numerical Procedure}

Numerical solutions of the governing equations with the associated boundary conditions were obtained using the control volume procedure outlined by Patankar [18]. An iterative procedure based on the SIMPLER algorithm was adopted for the momentum equations in the entrance region to link the pressure drop and the velocity field. By using this method, for any given thickness of the porous layer, equations (1), (2) and (3) were discretized and solved to attain the steady state solution. As a result, velocity and pressure drop in the fully developed region 
have been determined, Temperature field $\Theta$ was computed subsequently from the energy equation (11).

Momentum equations (2) ad (3) can be written in the flux form as

$\frac{\partial \phi}{\partial t}+\frac{\partial J_{X}}{\partial X}+\frac{1}{R} \frac{\partial J_{R}}{\partial R}=S$.

where $\phi$ stands for transport variables $U$ and $V . J_{X}$ and $J_{R}$ are the total (convection plus diffusion) fluxes defined by

$$
J_{X}=U \phi-\Gamma \frac{\partial \phi}{\partial X}, \quad J_{R}=R V \phi-\Gamma R \frac{\partial \phi}{\partial R},
$$

in which the diffusion coefficient is given by $\Gamma=\frac{2}{\mathrm{Re}}$.

$S$ is the sum of all source terms including pressure drop, Darcy and Forchheimer terms and the additional part from the diffusion term. Thus, for the momentum equation in the flux form in axial direction $S$ is given by

$$
S=-\frac{\partial P}{\partial X}-\varsigma\left[\frac{2 \varepsilon}{D a \operatorname{Re}}+\frac{\varepsilon^{2} C_{F}}{\sqrt{D a}}\left|\vec{V}_{D}\right|\right] U
$$

and in radial direction,

$$
S=-\frac{\partial P}{\partial R}-\varsigma\left[\frac{2 \varepsilon}{D a \operatorname{Re}}+\frac{\varepsilon^{2} C_{F}}{\sqrt{D a}}\left|\vec{V}_{D}\right|\right] V-\frac{2}{\operatorname{Re}} \frac{V}{R^{2}}
$$

There are many expressions for the linearization of source terms. In the present study, the form recommended by Patankar [18] was applied. That is,

$$
S=S^{*}+\left(\frac{d S}{d \phi}\right)^{*}\left(\phi_{p}-\phi_{p}^{*}\right),
$$

where superscript*denotes the guessed value or the previous-iteration value and subscript on $\phi$ indicates the main grid point labeled as $p$. Accordingly, the source term of the momentum equation in axial direction is linearized as

$S=S_{c}+S_{p} U_{p}$,

in which

$$
S_{c}=\varsigma \frac{\varepsilon^{2} C_{F}}{\sqrt{D a}} \frac{U_{p}^{*^{3}}}{\left|\sqrt{U_{p}^{*^{2}}+V_{p}^{*^{2}}}\right|}
$$

and

$$
S_{p}=-\varsigma\left\{\begin{array}{l}
\frac{2 \varepsilon}{D a \operatorname{Re}}+\frac{\varepsilon^{2} C_{F}}{\sqrt{D a}} \\
{\left[\frac{U_{p}^{*^{2}}}{\sqrt{U_{p}^{*^{2}}+V_{p}^{*}}}+\left|\sqrt{U_{p}^{*^{2}}+V_{p}^{*^{2}}}\right| \mid\right.}
\end{array}\right\}
$$

The control volume equations in the entrance developing region, form a set of linear (or at least nominally) simultaneous equations where $\phi$ values are unknowns and must be solved by iteration. A combination of lineby-line and block-correction methods was used to solve the resulting algebraic equations $[19,20]$.

For each grid point, a residual $\operatorname{Re} s$ can be calculated from the resulting discretized equation as

$\operatorname{Re} s=\sum a_{n b} \phi_{n b}+b-a_{p} \phi_{p}$,

where $\sum a_{n b} \phi_{n b}$ shows all neighbor terms, $a_{p}$ is the main grid point coefficient and $b$ is source term. The convergence criterion adopted was that $|\operatorname{Re} s|$ for each grid point, between two successive iterations were smaller than a pre-assigned value of $10^{-4}$.

The number and distribution of grids required in order to obtain acceptable accuracy were found by trial and error. For forced convection in porous media, it has been shown that solutions of the governing equations exhibit steep velocity gradientsuse near the impermeable walls due to imposing no-slip conditions at solid surfaces, and these gradients vary with the Darcy number, particle/pore diameter, Reynolds number and Prandtl number $[21,22]$. Consequently, a skewed grid distribution is used along all the coordinate axes such that the node density is higher in the vicinity of the wall and near the fluid/porous interface in the transverse direction and near the entrance in the longitudinal direction. The spatial grid was typically $67 \times 77$ in the $X-R$ computational domain, which consisted of the half-width pipe. Because this problem is strongly parabolic, the computational domain was systematically extended in the axial direction beyond the physical domain until the effects of exit boundary conditions on the flow in the entrance region were negligible. A similar staggered computational grid in a square driven cavity was used by Shokouhmand and Sayehvand [23].

The linearized forms of the governing equations in the fully-developed region can be summarized as

$a_{P} \phi_{P}=a_{N} \phi_{N}+a_{S} \phi_{S}+b$,

where $\phi$ stands for the transport variables: $U$ for the momentum equation and $\Theta$ for the energy equation; $a$ are coefficients combining diffusion terms and the nonconstant parts of the linearized Darcy and Forchheimer terms; and $b$ is sum of all source terms including pressure drop, the constant parts of linearized Darcy and Forchheimer terms and the additional part due to the temporal discretization. The subscripts on $a$ denote the main grid point labeled as $P$ surrounded by the two neighboring points labeled as $N$ (north) and $S$ (south). A matrix solver based on the Tri-Diagonal Matrix Algorithm (TDMA) was applied to solve the resulting discretized algebraic equations to attain the steady state results. The computational grid that is shown in Fig. 2 


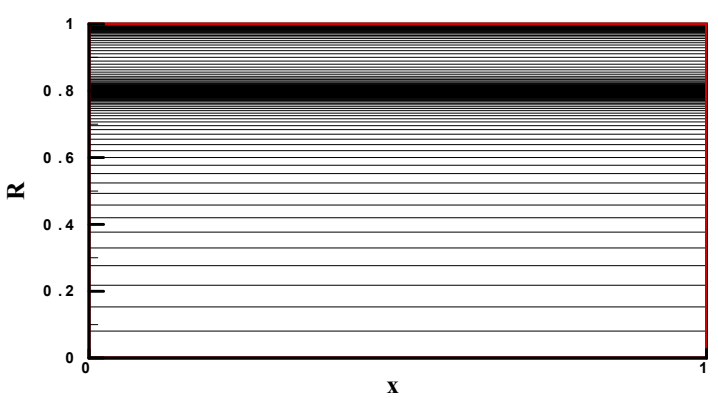

Fig.2. Computational grid for the fully developed region, $S=0.2$.

was used in the fully-developed region, and the number of grid points was 101 for the transverse direction

A harmonic-mean formulation was adopted for the interface diffusion coefficients between two control volumes, and this approach is capable of handling abrupt changes in these coefficients at the

fluid/porous interface. This approach is explained extensively by Patankar [18].

Several trial calculations were repeated to test the sensitivity of the results to grid size and time interval. The outcome of these exercises was satisfactory. In an effort to perform explicit comparisons with the previous studies [6], it was set that $\operatorname{Re}=50$ and $\operatorname{Pr}=7.0$ in the present study. This relatively low value of $\mathrm{Re}$ was chosen to simulate a reasonably slow slug flow [24].

\section{Results and Discussion}

Results of flow field and heat transfer are presented in terms of pressure drop, velocity profiles and Nusselt numbers. The effects of parameters based on physical properties of the porous material such as Darcy number ( $D a$ ) and the thermal conductivity ratio $\left(R_{k}\right)$ are discussed. The effect of the porous layer thickness, varying from $0 \%$ to $100 \%$ of the pipe radius, is also considered. The calculation domain is set as $0 \leq S \leq 1.0,10^{-4} \leq D a \leq 10^{-1}$ and $1.0 \leq R_{k} \leq 200.0$.

Entrance region for different Darcy numbers and thicknesses of the porous layer have been solved and pressure drops in the fully developed flow are obtained from these solutions. For the case of fully developed flow of a Newtonian fluid in a nonporous pipe, the result for pressure drop obtained in the present study was compared with the analytical solution of Poiseuille [25]. Very good agreement was found, which indicates validity of the code that has been developed.

In designing heat exchangers or regenerators, the primary goal is to minimize the mechanical loss due to pressure drop and to enhance the heat transfer rate from

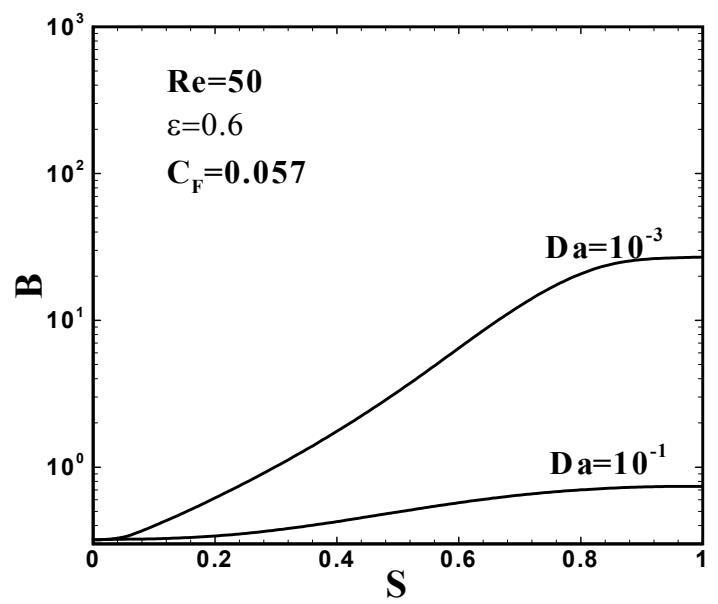

Fig.3. a. Pressure drop as a function of the thickness of porouslayer $D a=10^{-3}, D a=10^{-1}$.

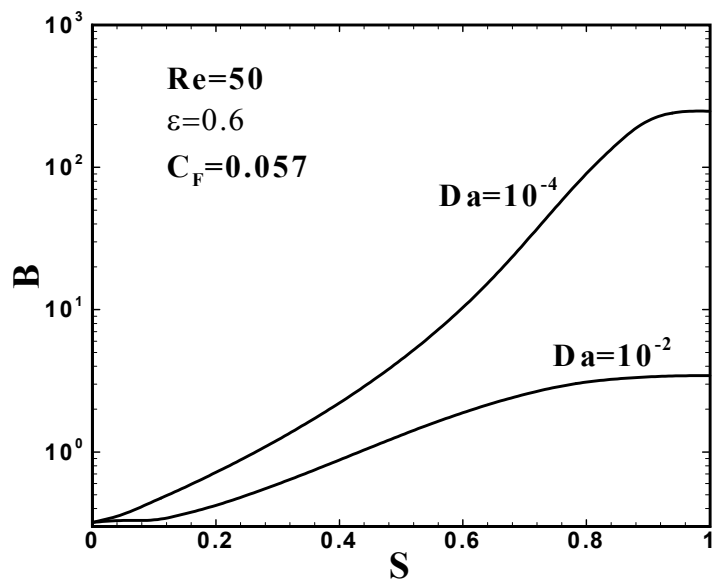

Fig.3. b. Pressure drop as a function of the thickness of porous layer $D a=10^{-4}, D a=10^{-2}$.

and to the working fluid. It is, therefore, important for the designer to compromise the tradeoff between pressure drop and heat transfer. One major motivation of the present study is to reduce pressure drop by employing a pipe partially filled with a porous medium instead of full porous medium. In an effort to demonstrate the characteristics of the pressure drop against the Darcy number and the porous layer thickness, the variation of $B=-(\partial P / \partial X)_{s}$ is shown in Fig. 3. The otherconditions are fixed at $\mathrm{Re}=50$, $\varepsilon=0.6$, and $C_{F}=0.057$. It is seen that, as $S$ increases or $D a$ decreases, $B$ increases. This means that the resistance of porous matrix to the flow becomes large, as $D a$ decreases or $S$ increases. Special attention is given to case $\left(D a=10^{-4}\right)$, the pressure drop $(B)$ is substantially increased, i.e. $B=248$ at $S=1$. This can 


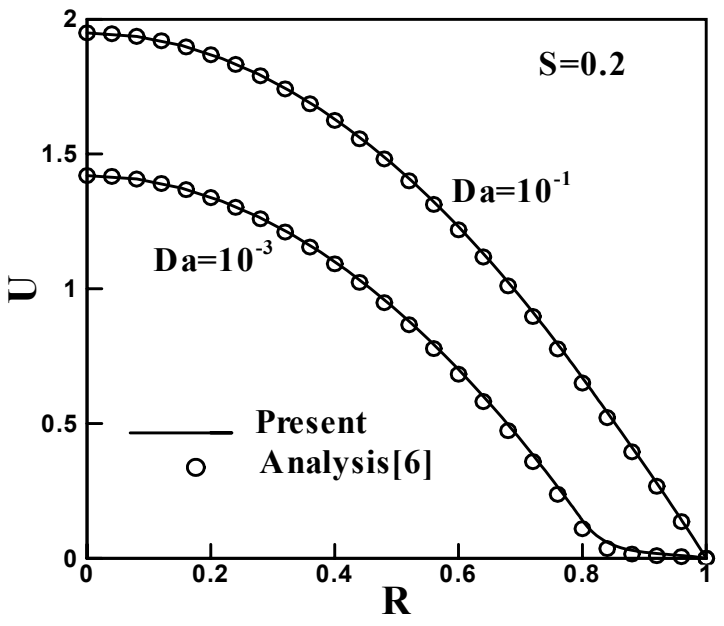

Fig.4. a. Comparison of velocity profile, $S=0.2$, $B=0.32, D a=10^{-3}, D a=10^{-1}$.

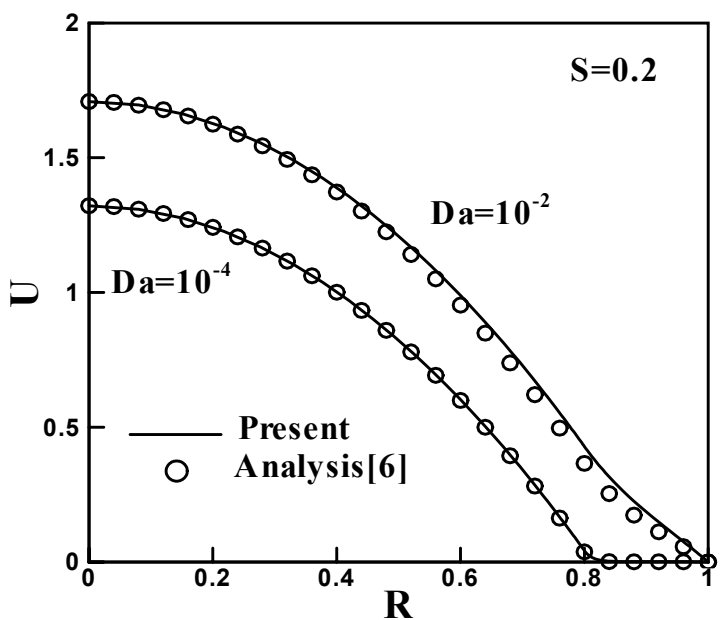

Fig.4. b. Comparison of velocity profile, $S=0.2$,

$$
B=0.32, D a=10^{-4}, D a=10^{-2} \text {. }
$$

be compared with $B=0.32$ at $S=0$. However, when $S \leq 0.5$, the pressure drop is mildly affected by $D a$.

In order to examine the validity and accuracy of the present numerical model in the fully developed flow, a comparative example is displayed in Fig. 4. It corresponds to the case where the thickness of porous layer near the wall equals $20 \%$ of the pipe radius. The pressure drop remains constant $(B=0.32)$. In accordance with the analysis [6], the Forchheimer term of porous material is not accounted for $\left(C_{F}=0\right)$. It is seen that the present numerical results are in excellent agreement with the analytical solution of Poulikakos and Kazmierczak [6]. This exemplifies the reliability of the

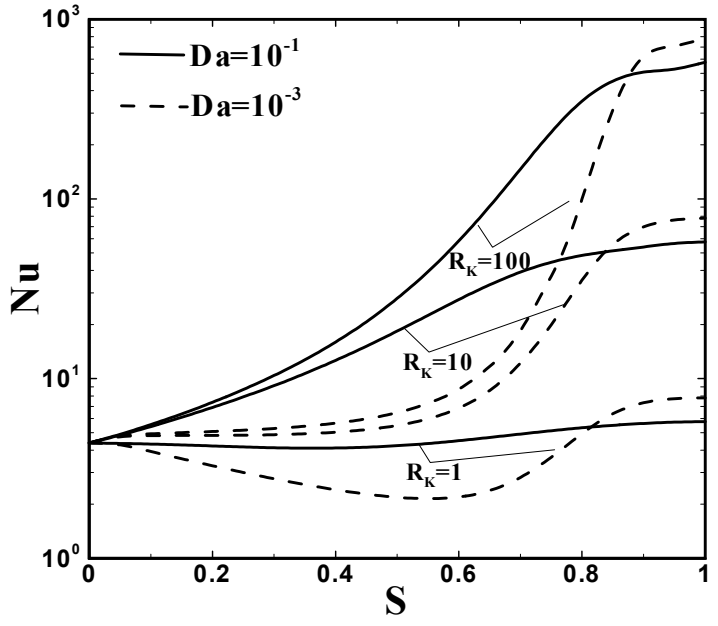

Fig.5. a. Variations of Nusselt number as a function of the thickness of porous layer, $D a=10^{-3}, D a=10^{-1}$

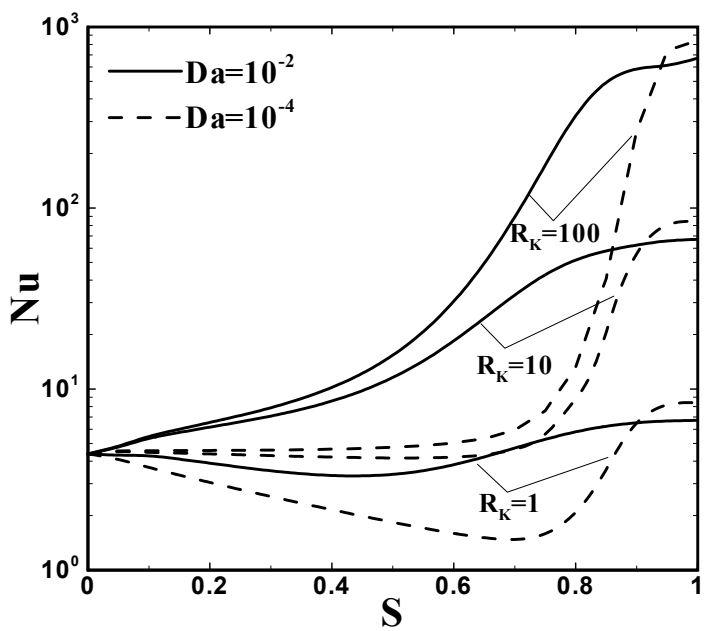

Fig.5. b. Variations of Nusselt number as a function of the thickness of porous layer, $D a=10^{-4}, D a=10^{-2}$.

present simulation.

Processing the numerical results of the temperature field, the heat transfer properties are now examined. In order to compare heat transfer results to the case where no porous medium is present, the Nusselt number is redefined with respect to the thermal conductivity of the fluid. In the case of constant wall heat flux, the Nusselt number is generally defined as

$N u=\frac{2}{\Theta_{w}-\Theta_{b}}$

in which $\Theta_{w}$ denotes the non-dimensional temperature at the wall and $\Theta_{b}$ is the non-dimensional bulk temperature. The Nusselt number is plotted in Fig. 5 against the porous layer thickness $(0 \leq S \leq 1)$, and the thermal 
conductivity ratio $\left(R_{k}=1,10\right.$ and 100$)$. Four cases of permeability are also selected for comparison: $D a=10^{-1}, 10^{-2}, 10^{-3}$ and $10^{-4}$. As seen in these figures, the dependence of $N u$ on $S$ is not straightforward. In the case of a low thermal conductivity material $\left(R_{k}=1\right)$ which may be used for insulation, for a given permeability, the Nusselt number decreases when the porous layer thickness increases, up to a critical value beyond which, $N u$ increases to end. The physical explanation is that, when the porous layer thickness increases, the flow rate is reduced and hence the prescribed heat flux makes the wall temperature increase more than the mean temperature of the fluid. The Nusselt number, being inversely proportional to the temperature difference, decreases until the critical thckness is reached. Over this value, the inverse effect is produced, that is, the fluid mean temperature increases more than the wall temperature and thus $N u$ is augmented. Similar results were shown by Poulikakos and Kazmierczak [6] for a partially filled cylinder, by Lauriat and Vafai [26] for a parallel plate channel, by Campos et al. [27] and by others [28, 29]. The effect of permeability is also seen in these figures through the different values of $D a$. The more permeable is the medium, the higher is heat transfer and the lower is the critical thickness. One can deduce that there is no need to fill up the pipe with the porous material to obtain the minimum heat transfer.

The critical thickness disappears as the porous material becomes more conducting, for any permeability, as shown in Fig. 5, and the Nusselt number increases monotonically with increasing $S$. Beyond a certain thermal conductivity value, which varies with the permeability, the presence of the porous medium enhances heat transfer. In this case, because of the presence of high conducting porous material, the imposed heat flux rapidly transfers to the fluid and hence $N u$ is increased. The Nusselt number increases substantially, notably for a highly conducting material ( $R_{k}=100$ ), up to nearly a constant value which is reached when approximately $90 \%$ of the pipe is filled (for $D a=10^{-1}$ ). If the medium is more permeable, a lower $N u$ is obtained and the constant value is reached for smaller thickness. Thus, even with a highly conducting material, it is sufficient to fill the pipe just to a critical thickness to reach the maximum heat transfer rate as shown in Fig. 5.

The Nusselt number is plotted in Fig. 6 against the thermal conductivity ratio, and the Darcy number for two cases of the thicknesses of porous layer, i.e. $S=0.2$ and $S=0.95$. It is seen that, for the lower values of $S$, by increasing $D a, N u$ increases and the

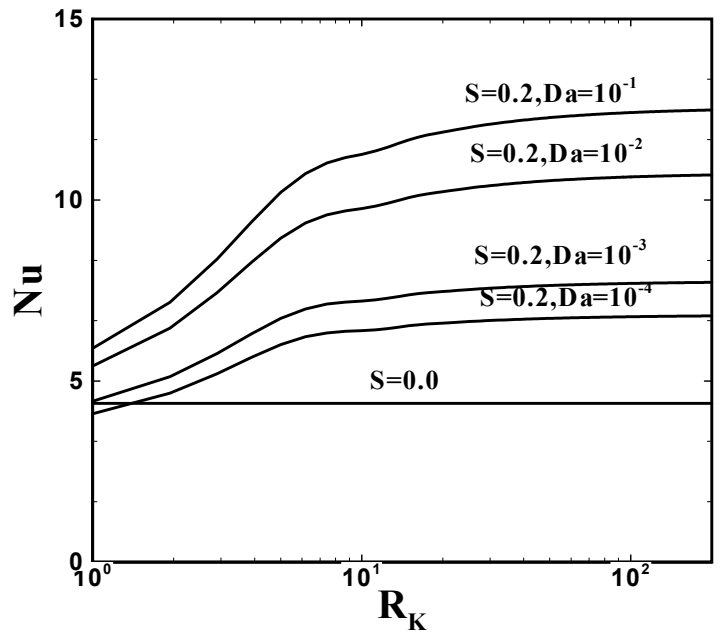

Fig.6. a. Variations of Nusselt number vs thermal conductivity ratio for different Darcy numbers, $S=0.2$.

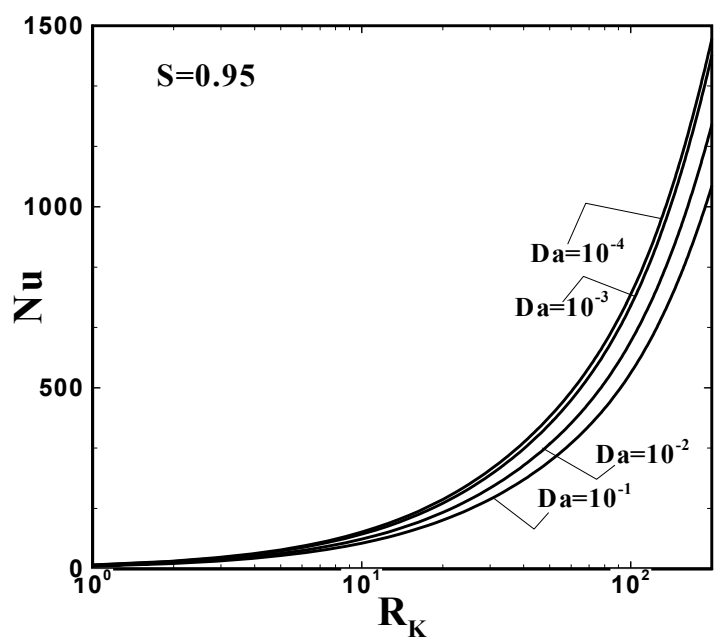

Fig.6. b. Variations of Nusselt number vs thermal conductivity ratio for different Darcy numbers, $S=0.95$.

inverse effect is observed for the higher values of $S$. Further, in Fig. 6.a, the role played by the porous layer with respect to heat transfer is shown. For a relatively low permeability $\left(D a=10^{-4}\right)$, the porous medium present a resistance to heat transfer, unless the material is nearly 1.6 as much as the fluid $\left(R_{k}=1.6\right)$, beyond which point the medium enhances heat transfer. For a higher permeability material, it happens even at the lower thermal conductivity ratio.

These findings are qualitatively in conformity with the analysis of an annular duct partilly filled with a porous medium by Chikh et al. [8]. 


\section{Conclusion}

Detailed numerical analysis is done in a circular pipe partially filled with a porous medium to delineate the flow and heat transfer characteristics. The presence of a porous matrix is shown to present a resistance to the flow and heat transfer for low permeability materials. However, for highly conducting porous media, heat transfer is systematically augmented whatever the $D a$ value is. It is also shown, whether the porous material is used for insulation or for enhancement of heat transfer, there is no need to fill the pipe completely, especially if the medium is highly permeable.

\begin{tabular}{|c|c|}
\hline \multicolumn{2}{|l|}{ Nomenclature } \\
\hline$a$ & pipe radius \\
\hline b & source term \\
\hline$B$ & $\begin{array}{l}\text { steady non-dimensional axial } \\
\text { pressure drop, }-\left(\frac{\partial P}{\partial X}\right)_{s}\end{array}$ \\
\hline$C_{F}$ & $\begin{array}{l}\text { inertia coefficient of porous } \\
\text { medium }\end{array}$ \\
\hline$C_{p}$ & specific heat capacity of fluid \\
\hline$D a$ & Darcy number, $K / a^{2}$ \\
\hline$J_{R}, J_{X}$ & total fluxes \\
\hline$k$ & thermal conductivity of fluid \\
\hline$K$ & $\begin{array}{l}\text { permeability of porous } \\
\text { medium }\end{array}$ \\
\hline$N u$ & Nusselt number \\
\hline$p, P$ & $\begin{array}{l}\text { pressure, non-dimensional } \\
\text { pressure }\end{array}$ \\
\hline $\operatorname{Pr}$ & Prandtl number \\
\hline$q "$ & heat flux \\
\hline$r, x$ & dimensional coordinates \\
\hline$R, X$ & non-dimensional coordinates \\
\hline$R_{c}$ & ratio of heat capacity of porous \\
\hline$R_{k}$ & $\begin{array}{l}\text { medium to fluid } \\
\text { thermal conductivity ratio }\end{array}$ \\
\hline $\operatorname{Re}$ & Reynolds number \\
\hline $\operatorname{Re} s$ & residual \\
\hline$s, S$ & $\begin{array}{l}\text { dimensional, non-dimensional } \\
\text { thickness of porous layer } \\
\text { (source term) }\end{array}$ \\
\hline$t$ & non-dimensional time \\
\hline$T$ & dimensional temperature \\
\hline$T_{\infty}$ & inlet temperature \\
\hline$u, v$ & $\begin{array}{l}\text { dimensional velocity } \\
\text { components }\end{array}$ \\
\hline
\end{tabular}
$U, V$
non-dimensional velocity
components
$u_{0} \quad$ inlet velocity
$\vec{V}_{D}$
Darcian velocity vector

\section{Greek symbols}

$\alpha \quad$ thermal diffusivity of fluid

$\varepsilon \quad$ porosity of porous medium

$\phi \quad$ transport variable

$v \quad$ kinematic viscosity

$\rho \quad$ density

$\tau \quad$ dimensional time

$\Gamma \quad$ diffusion coefficient

$\Theta \quad$ non-dimensional temperature

$\begin{array}{ll}\text { Subscripts } & \\ b & \text { bulk } \\ \text { eff } & \text { effective value of porous } \\ & \text { medium } \\ s & \text { steady component } \\ w & \text { wall }\end{array}$

References:

[1] Nield, D. A. and Bejan, A. (1992) Convection in porous media, Springer-verlag, New York, NY.

[2] Kaviany, M. (1995), Principles of Heat Transfer in Porous Media, Springer-Verlag, New York, NY.

[3] Beckermann, C., Ramadhyani, S., and Viskanta, R. (1986), Natural convection flow and heat transfer between a fluid layer and a porous layer inside a rectangular enclosure, AIAA/ASME Heat Transfer and Thermophysics Conference, Boston, MA

[4] Somerton, C. W. and Catton, I., (1982), On the thermal instability of superposed porous and fluid layers, ASME Journal of Heat Transfer, Vol. 104, pp. 160-165.

[5] Nield, D. A. (1977), Onset of convection in a fluid layer overlaying a layer of porous medium, Journal of Fluid Mechanics, Vol. 81, pp. 513-522.

[6] Poulikakos, D. and Kazmierczak, M. (1987), Forced convection in a duct partially filled with a porous material, Trans. ASME Journal of Heat Transfer, Vol. 109, pp. 653-662.

[7] Tong, T. W. and Sharatchandra, M. C. (1990), Heat Transfer enhancement using porous inserts, Heat Transfer and Flow in Porous Media, HTD 156. pp. 4146.

[8] Chikh, S., Boumedien, A., Bouhadef, K. and Lauriat, G. (1995), Analytical solution of non-Darcian forced convection in an annular duct partially filled with a porous medium, International Journal of Heat and Mass Transfer, Vol. 38, pp. 1543-1551. 
[9] Sung, H. J., Kim, S. Y. and Hyun, J. M. (1995), Forced convection from an isolated heat source in a channel with porous medium, International Journal of Heat and Fluid Flow, Vol. 16, pp. 527-535.

[10] Guo, Z., Kim, S. Y. and Sung, H. J. (1997), Pulsating flow and heat transfer in a pipe partially filled with a porous medium, International Journal of Heat and Mass Transfer, Vol. 40, No. 17, pp. 4209-4218.

[11] Vafai, K. and Tien, C. L. (1981), Boundary and inertia effects on flow and heat transfer in porous media, International Journal of Heat and Mass Transfer, Vol. 24, pp. 195-203.

[12] Lauriat, G. and Prasad, V. (1989), Non-Darcian effects on natural convection in a vertical porous enclosure, International Journal of Heat and Mass Transfer, Vol. 32, pp. 2135-2147.

[13 Kladias, N. and Prasad, V. (1990), Flow transitions in buoyancy-induced non-Darcy convection in a porous medium heated from below, Trans. ASME Journal of Heat Transfer, Vol. 112, pp. 675-684.

[14] Khodadadi, J. M. (1991), Oscillatory fluid flow through a porous medium channel bounded by two impermeable parallel plates, Journal of Fluid Engineering, Vol. 113, pp. 509-511.

[15] Kim, S. Y., Kang, B. H. and Hyun, J. M. (1994), Heat transfer from pulsating flow in a channel filled with porous media, International Journal of Heat and Mass Transfer, Vol. 37, pp. 2025-2033.

[16] Lundgren, T. S. (1972), Slow flow through stationary random beds and suspension of spheres, Journal of Fluid Mechanics, Vol. 51, pp. 273-299.

[17] Chatwin, P. C. (1975), On the longitudinal dispersion of passive contaminant in oscillatory flows in tubes, Journal of Fluid Mechanics, Vol. 71, pp. 513-527.

[18] Patankar, S. V. (1980), Numerical Heat Transfer and Fluid Flow, Hemisphere, New York.

[19] Settari, A. and Aziz, K. (1973), A generalization of the additive correction methods for the iterative solution of matrix equations, SIAM J. Numer, Anal., Vol. 10, pp. 506-521.

[20] Johnston, R. W. (editor) (1998), The Handbook of Fluid Dynamics, Springer and CRC Press.

[21] Cheng, P., Hsu, C. T. and Chowdhury, A. (1988), Forced convection in the entrance region of a packed channel with asymmetric heating, ASME Journal of Heat Transfer, Vol. 110, pp. 946-054.

[22] Hunt, M. L. and Tien, C. L. (1988), Effects of thermal dispersion on forced convection in fibrous media, International Journal of Heat and Mass Transfer, Vol. 31, pp. 301-309.

[23] Shokouhmand, H. and Sayehvand, H. (2004), Numerical study of flow and heat transfer in a square driven cavity, International Journal of Engineering, Vol. 17, No. 3, pp. 301-317.
[24] Siegel, R. and Perlmutter, M. (1962), Heat transfer for pulsating laminar duct flow, Trans. ASME Journal of Heat Transfer, Vol. 84, pp. 111-123.

[25] O'Neill, M. E. and Charlton, F. (1989), Viscous and Compressible Fluid Dynamics, Ellis Harwood Limited, England.

[26] Lauriat, G. and Vafai, K. (1991), Forced convection flow and heat transfer through a porous medium exposed to a flat plate or a channel. In Convective Heat and Mass Transfer in Porous Media, NATO ASI Series, Series E, Vol. 196, pp. 289-327, Kluwer Academic Publisher.

[27] Campos, H., Morales, J. C., Lacoa, U. and Campo, A. (1990), Thermal aspects of a vertical annular enclosure divided into a fluid region and a porous region, Int. Commun. Heat Mass Transfer, Vol. 17, pp. 343-354. [28] Tong, T. W. and Subramanian, E. (1983), Natural convection in rectangular enclosure partially filled with a porous medium, Proc. ASME/JSME Thermal Engineering Joint Conference, Vol. 1, pp. 331-338.

[29] Prasad, V., Brown, K. and Tian, Q. (1989), Flow visualization and heat transfer experiments in fluid superposed porous layers heated from below, ASME, Winter Annual Meeting. 